\title{
Prospecting for Precision: Promises for Personalized Medicine
}

In this issue of The Journal, co-first authors Kim, Bang, and colleagues present provocative data suggesting an association with the clinical response to cyclophosphamide (CYC) therapy in patients with active lupus nephritis: whether administered according to the US National Institutes of Health (NIH) regimen of monthly intravenous doses or according to the Euro-Lupus biweekly schedule, response to CYC is associated with single-nucleotide polymorphism (SNP) in the telomeric end of the FCGR gene cluster adjacent to $F C G R 2 B$ on chromosome $1 \mathrm{q} 23^{1}$. The $F C G R 2 B$ gene encodes the cell surface receptor, FcyRIIb (CD32B), which is the only Fcy receptor with a tyrosine-based inhibitory motif in the human genome. The prospect of genetically based personalized, precision medicine coming to rheumatologic conditions apart from certain autoinflammatory and rare disease states ${ }^{2}$ and specific considerations in pharmacogenomics ${ }^{3}$ is very appealing and holds high promise for the future. The reasonable question is whether we have arrived or have more to do.

The study by Kim and Bang's group defines complete, partial, and non-responsiveness to CYC and relates these clinical categories to nearly 500,000 SNP in germline DNA obtained in a genome-wide association study. The technology is powerful and continues to advance with more densely featured SNP genotyping arrays and even with whole-genome sequencing, which is now both technically and financially within reach. Thus, the study design often determines the power of any given project, and Kim and Bang's group points out that their study population is relatively small and that there is no replication to confirm findings. Fine mapping, in large part through imputation, suggests local support for the association of clinical non-responsiveness with an SNP at the 3' end of FCGR2B (rs6697139), but the availability of other directly genotyped markers in the region is limited. This is not a surprise because the human FCGR locus is characterized by a distant duplication event, compounded by both additional deletions and duplications within human populations $s^{4,5}$.

Nonetheless, the finding of an association of an SNP just 3 ' of the FCGR2B coding region is intriguing. Fc receptors encoded in this region of the genome bind IgG antibodies and are integral to the handling of immune complexes, whether for disposal or for cell signaling with activation of cell programs in myeloid cells, platelets, B lymphocytes, natural killer cells, and even some $\mathrm{T}$ lymphocytes ${ }^{6}$. In addition to pathways activated through $\mathrm{Fc}$ receptors per se, the cell signaling pathways initiated in response to nucleic acid-containing immune complexes may result from cooperative signals between Toll-like receptors and Fc receptors. Such cooperative signaling may also engage complement receptors after fixation of complement by the immune complexes; and based on the antigen in the immune complexes, it is likely that other receptor systems can be engaged both on the surface and in the cytoplasm of the cell.

Predicting the effect of an SNP such as rs6697139 can be challenging. The effect of a nonsynonymous coding SNP, which changes the protein sequence, can be approached with various bioinformatics algorithms ${ }^{7}$. Such protein coding variants have been identified and studied among members of the human low affinity Fc receptor cluster; and variants affecting ligand binding affinity ${ }^{8}$, glycosylation sites $^{9}$, lateral mobility in the plane of the cell membrane ${ }^{10}$, and signaling potential ${ }^{11}$ have been identified. Variants affecting expression include not only regulatory SNP but also stop codons in coding sequences that affect expression by causing premature termination ${ }^{12}$. Studies of putative regulatory SNP are most straightforward when they are in the proximal promoter ${ }^{13}$, and more difficult when they are in more distal sites. It is possible that CYC is affecting FCGR2B expression through an effect mediated by the rs6697139 major allele; but the work of Palmero, et al ${ }^{14}$, referred to by the Kim and Bang group, suggests augmentation of receptor binding and with increased functional activity with CYC administration rather than the inhibition of function one might anticipate with increased expression (and binding function) of the inhibitory FcyRIIb. Despite this ambiguity, regulatory SNP are likely to play an important role in the genetic architecture of autoimmune phenotypes ${ }^{15}$; and the understanding of how they contribute to disease risk and inform precision medicine is an exciting challenge for future investigation.

The goal of precision medicine is often phrased as the

See GWAS for CYC response, page 1045

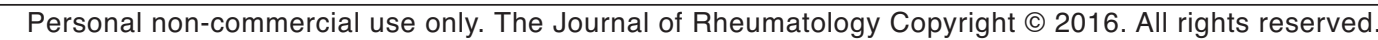


right treatment for the right patient at the right time. It has been embraced by many research initiatives and agencies including the European Union's Innovative Medicine Initiative 2 (www.euresearch.ch/en/european-programmes/further-programmes/jtis/innovative-medicines-initiative/), the Canadian Institutes of Health Research Signature Initiative (www.cihr-irsc.gc.ca/e/43627.html), and the NIH's Precision Medicine Initiative program (www.nih.gov/precision-medicine-initiative-cohort-program), and holds the promise of more effective healthcare and better health. It also anticipates a deeper understanding of both individual and population-based differences in disease manifestations and disease management, based on genetic background, environment, and lifestyle. Might there be some such clues about lupus nephritis in this work by Kim and Bang's group?

It is interesting to note that rs 1050501 , a nonsynonymous SNP at position 161643798 in the genomic region encoding the transmembrane segment of FcyRIIb (Ile232Thr), is a strong risk factor for systemic lupus in multiple East Asian populations, but not in white populations ${ }^{16,17}$. It lies between rs1771568 and rs10917686 (see Table $2^{1}$, Kim and Bang) and might be involved in the proposed association of rs6697139 with therapeutic response. Such an involvement could reflect rs6697139 serving as a surrogate marker for rs 1050501 through linkage disequilibrium. Alternatively, both SNP might influence distinct and synergistic biological properties, or there may be some other epistatic relationship reflecting ancestral background. The insight, and perhaps the caution, could be that the proposed association of rs6697139 with the clinical response of lupus nephritis to CYC therapy may be reproducible in East Asians but not in other ancestry groups, just as the rs 1050501 association with lupus risk is most strongly found in East Asian groups but only weakly in other population groups.

Thus, the answer to the question initially posed is that we have not yet arrived and we have more to do. The concept of personalized, precision medicine has generated a great deal of interest since genomic technology matured to the point of making genetic contributions to the matrix of precision achievable. Nonetheless, the prospect generates controversy ${ }^{18}$, and we can anticipate that the pages of The Journal and other forums will serve as sounding boards for new applications and approaches to "big data," and potentially for our approaches to clinical trials ${ }^{19}$ as we develop a deeper understanding of both individual and population-based differences in disease manifestations and disease management.

\section{ROBERT P. KIMBERLY, MD;} Howard L. Holley Professor of Medicine, Director, University of Alabama at Birmingham Center for Clinical and Translational Science, University of Alabama at Birmingham, Division of Immunology and Rheumatology, 1530 3rd Avenue South,

Birmingham, Alabama 35294-2182, USA. Address correspondence to Dr. R.P. Kimberly; E-mail: rpk@uab.edu

\section{REFERENCES}

1. Kim K, Bang SY, Joo YB, Kim T, Lee HS, Kang C, et al. Response to intravenous cyclophosphamide treatment for lupus nephritis associated with polymorphisms in the FCGR2B-FCRLA locus J Rheumatol 2016;43:1045-9.

2. Ombrello MD, Sikora KA, Kastner DL. Genetics, genomics and their relevance to pathology and therapy. Best Pract Res Clin Rheumatol 2014;28:175-89

3. Bush WS, Crosslin DR, Obeng AO, Wallace J, Almoquera B, Basford MA, et al. Genetic variation among 82 pharmacogenes: The PGRN-Seq data from the eMERGE Network. Clin Pharmacol Ther 2016 Feb 9 (E-pub ahead of print).

4. Su K, Wu J, Edberg JC, McKenzie SE, Kimberly RP. Genomic organization of classical low-affinity Fc-gamma receptor genes. Genes Immun 2002;3 Suppl 1:S51-6.

5. Mueller M, Barros P, Witherden AS, Roberts AL, Zhang Z, Schaschi $\mathrm{H}$, et al. Genomic pathology of SLE-associated copy-number variation at the FCGR2C/FCGR3B/FCGR2B locus. Am J Hum Genet 2013;92:28-40.

6. Brown EE, Edberg JC, Kimberly RP. Fc receptor genes and the systemic lupus erythematosus diathesis. Autoimmunity 2007;40:567-81.

7. Kircher M, Witten DM, Jain P, O'Roak BJ, Cooper GM, Shendure J A general framework for estimating the relative pathogenicity of human genetic variants. Nat Genet 2014;46:310-5.

8. Wu J, Edberg JC, Redecha PB, Bansal V, Guyre PM, Coleman K, et al. A novel polymorphism of FcgammaRIIIa (CD16) alters receptor function and predisposes to autoimmune disease. J Clin Invest 1997;100:1059-70.

9. Drescher B, Witte T, Schmidt RE. Glycosylation of FcgammaRIII in N163 as mechanism of regulating receptor affinity. Immunology 2003;110:335-40.

10. Li X, Wu J, Carter RH, Edberg JC, Su K, Cooper GS, et al. A novel polymorphism in the Fcgamma receptor IIB (CD32B) transmembrane region alters receptor signaling. Arthritis Rheum 2003;48:3242-52.

11. Wu J, Ji C, Xie F, Langefeld CD, Qian K, Gibson AW, et al. FcalphaRI (CD89) alleles determine the pro-inflammatory potential of serum IgA. J Immunol 2007;178:3973-82.

12. Li X, Wu J, Ptacek T, Redden DT, Brown EE, Alarcon GS, et al. Allelic-dependent expression of an activating Fc receptor on B cells enhances humoral immune responses. Sci Transl Med 2013;5:216ra175.

13. Su K, Wu J, Edberg JC, Li X, Ferguson P, Cooper GS, et al. A promoter haplotype of the immunoreceptor tyrosine-based inhibitory motif-bearing FcgammaRIIb alters receptor expression and associates with autoimmunity. I. Regulatory FCGR2B polymorphisms and their association with systemic lupus erythematosus. J Immunol 2004;172:7186-91

14. Palmero MS, Giordano M, Serebrinsky GP, Geffner JR, Ballart I, Isturiz MA. Cyclophosphamide augments ADCC by increasing the expression of Fc-receptors. Immunol Lett 1987;15:83-7.

15. Fahr KK-H, Marson A, Zhu J, Kleinewietfeld M, Housley W, Beik $\mathrm{S}$, et al. Genetic and epigenetic fine mapping of causal autoimmune disease variants. Nature 2015;518:337-43.

16. Tsuchiya N, Kyogohu C. Role of Fc $\gamma$ RIIb polymorphisms in the background of systemic lupus erythematosus: insights from Asia. Autoimmunity 2005;38:347-52.

17. Willcocks LC, Carr EJ, Niederer HA, Rayner TF, Williams TN, Yang W, et al. A defunctioning polymorphism in FCGR2B is associated with protection against malaria but susceptibility to systemic lupus erythematosus. Proc Natl Acad Sci USA 2010;107:7881-5.

18. Interlandi J. The paradox of precision medicine. Sci Am 2016;314:24-B24.

19. Schork J. Personalized medicine: time for one person trials. Nature 2015;520:609-11.

J Rheumatol 2016;43:999-1000; doi:10.3899/jrheum.160424

Personal non-commercial use only. The Journal of Rheumatology Copyright @ 2016. All rights reserved 